Gut, 1982, 23, 872-874

Case report

\title{
Massive gastrointestinal bleeding in Crohn's disease: successful control by intra-arterial vasopressin infusion
}

\author{
J A MELlOR,* G N CHANDLER, A H CHAPMAN, and H C IRVING \\ From Chapel Allerton Hospital, Leeds
}

SUMMARY A case of massive lower gastrointestinal bleeding from Crohn's disease is reported. This is an uncommon complication of the disease and the literature is reviewed. Successful control of gastrointestinal bleeding by intra-arterial vasopressin infusion has not previously been reported in Crohn's disease. We suggest that this technique may be used in an attempt to avoid surgery.

Massive lower gastrointestinal haemorrhage is an unusual complication of Crohn's disease. Homan $e t$ $a l^{1}$ recorded only seven cases in 43 years at the New York Cornell Medical Centre. Angiography may demonstrate the bleeding site and can be followed by resection of the diseased bowel. ${ }^{12}$ After one attack of massive bleeding, further episodes are likely. Among 29 similar cases reported there were 12 recurrences. ${ }^{1-3}$ Control of bleeding by selective intra-arterial infusion of vasopressin may be attempted, ${ }^{4}$ although Harvey $e t \mathrm{al}^{2}$ found no report of its successful use and considered that the potential hazards limited wider acceptance.

This is the first report of the successful use of intra-arterial vasopressin in controlling massive lower gastrointestinal bleeding in a patient with Crohn's disease.

\section{Case report}

A 32 year old woman with a 17 year history of small bowel Crohn's disease was admitted to Chapel Allerton Hospital because of abdominal pain and profuse watery diarrhoea. She had had two previous intestinal resections and the remaining metre of jejunum was anastomosed to ascending colon. Sigmoidoscopy showed the rectum and sigmoid colon to be affected. She was anaemic (haemoglobin

\footnotetext{
* Present address: Department of Clinical Medicine, TCD Medical School Building, St James's Hospital, James's Street, Dublin 8, Ireland.

Received for publication 26 January 1982
}

$11.0 \mathrm{~g} / \mathrm{dl}$ ) and dehydrated (urea $32.5 \mathrm{mmol} / \mathrm{l}$ ). Correction of fluid and electrolyte imbalance and administration of corticosteroids was associated with dramatic clinical improvement. Seven days after admission her recovery was interrupted by profuse rectal bleeding. During the following 24 hours, $4 \mathrm{l}$ of clot and dark red blood was passed, transfusion of 3.51 of blood being needed to maintain vital signs. Gastroduodenoscopy was normal and there was no evidence of a bleeding diathesis. Mesenteric angiography demonstrated extravasation of contrast from a branch of the left colic artery in the region of the splenic flexure (Fig. $1)$. The angiographic catheter was left in position and used to selectively infuse a solution of vasopressin (0.1 units/min) into the inferior mesenteric artery. Angiography was repeated after 20 minutes, demonstrating generalised arterial constriction with cessation of bleeding (Fig. 2). The infusion was changed to normal saline and the patient returned to the ward with the angiographic catheter in situ. Bleeding recurred 10 hours later but was quickly controlled by a further vasopressin infusion, which was, on this occasion, continued for 48 hours $(0.2$ units/min for 24 hours followed by 0.1 units/min for a further 24 hours) The catheter was removed without significant complication after four days. Subsequent colonoscopy confirmed colonic involvement, which was maximal at the splenic flexure. The patient has remained well, maintaining a normal blood count for six months to date. 


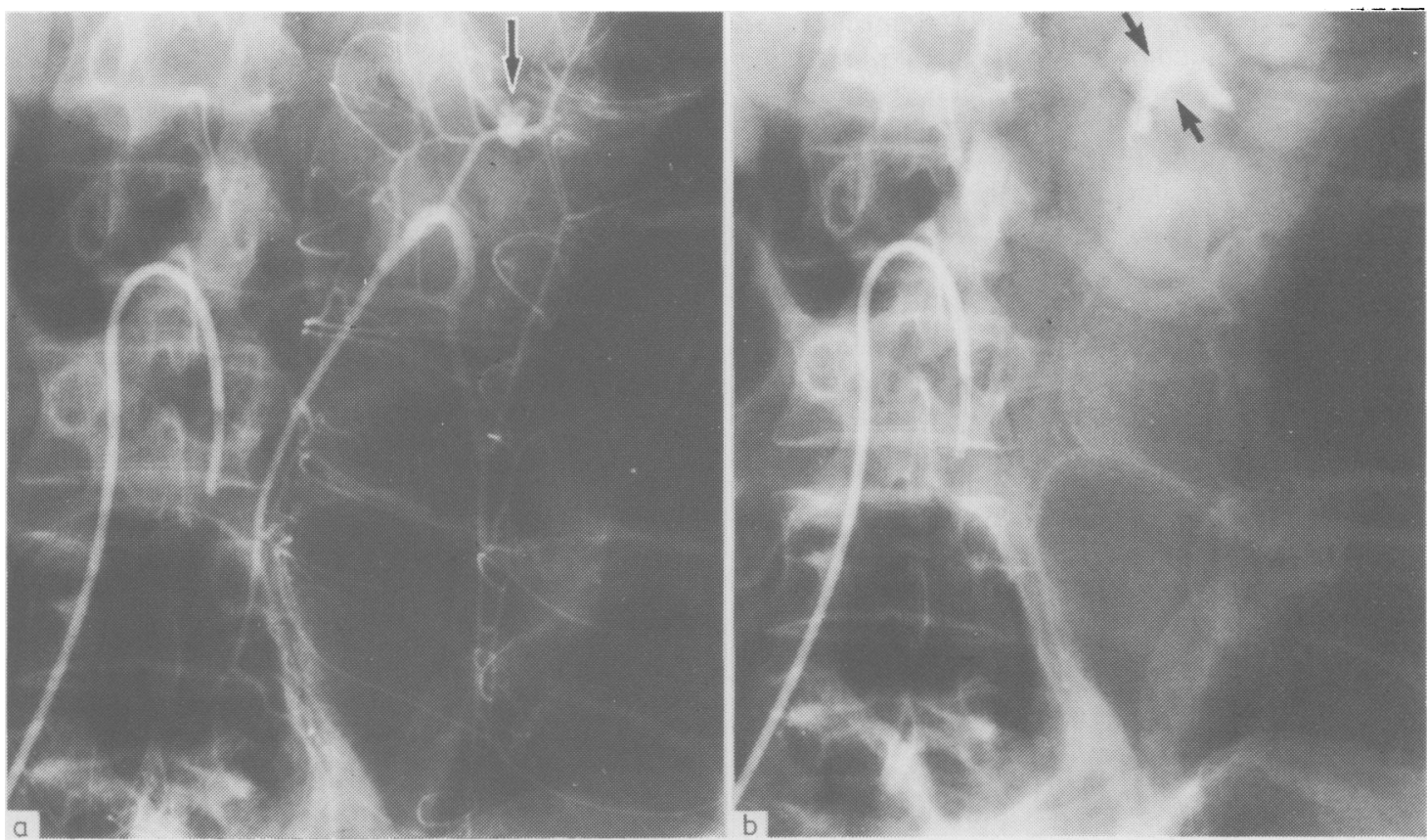

Fig. 1 Inferior mesenteric arteriogram: (a) Arterial phase film showing extravasation of contrast medium (arrows). (b) Venous phase film showing a crescent-shaped collection of contrast medium (arrows).

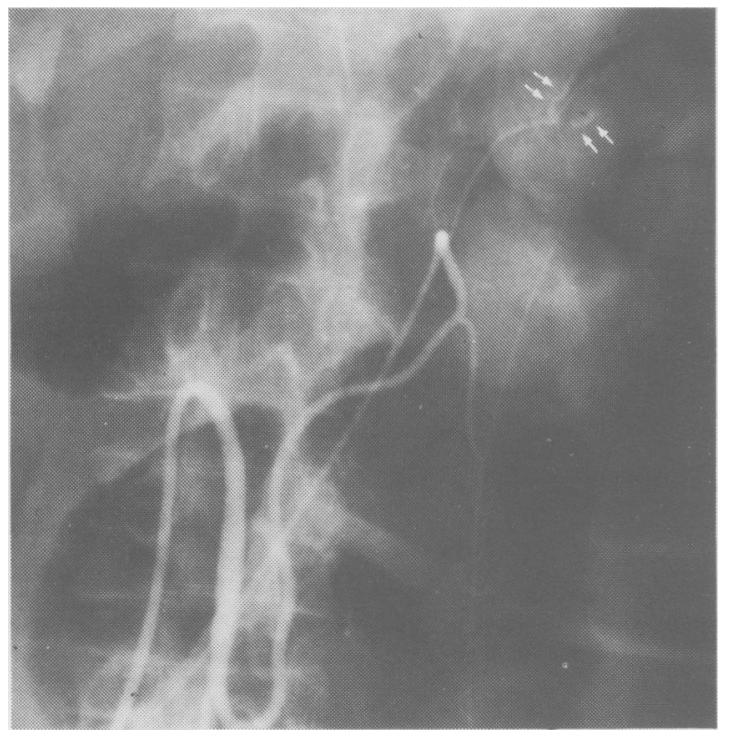

Fig. 2 Inferior mesenteric arteriogram following vasopressin infusion. Early arterial phase film showing generalised vasoconstriction. Two small arterial branches show calibre irregularities (arrows). No extravasation of contrast is seen.

\section{Discussion}

Endoscopy is the primary investigation for acute gastrointestinal haemorrhage but, if this fails to identify the bleeding site, diagnostic angiography may be performed, although bleeding at a rate of at least $0.5 \mathrm{ml} / \mathrm{min}$ is necessary for successful visualisation. ${ }^{5}$ With heavy bleeding sufficient contrast may extravasate to outline an ulcer or diverticulum or show the mucosal pattern of the bowel. In our patient, a crescent of contrast was seen on late films (Fig. 1b) which was identical to the 'pseudovein' described by Ring et $a l^{6}$ and attributed to extravasated contrast being contained by blood clot. At the site of extravasation, the post-infusion films showed two small arterial branches with calibre irregularities (Fig. 2). These vessels were presumed to be the origin of the bleed due to involvement in the inflammatory process.

Mesenteric arterial constriction in response to vasopressin infusion has been shown in early Crohn's disease. ${ }^{7}$ We were, however, cautious in our use of vasopressin, as it has been suggested that it might aggravate the intestinal ischaemia of established Crohn's disease and lead to bowel necrosis. ${ }^{2}$ In an attempt to avoid such problems, the initial vasopressin infusion was discontinued after 30 
minutes and angiography had demonstrated cessation of bleeding. When further bleeding occurred, however, a prolonged vasopressin infusion was used according to the regime recommended by Gordon et al. ${ }^{8}$ Our patient had a very long history of Crohn's disease, but colonic involvement was recent and could account for the excellent response which was seen.

Problems associated with angiographic control of intestinal bleeding include sepsis, arterial thrombosis, pseudo-aneurysm, and puncture site bleeding. ${ }^{8}$ All these are rare, with the exception of bleeding at the puncture site which can be easily controlled by manual compression. The pharmacological properties of vasopressin explain some of the complications of arterial infusion; bowel colic is common and can be minimised by slowly increasing the rate of infusion. Systemic effects such as myocardial ischaemia and fluid retention are unlikely to occur with the low dosage infused selectively in this manner.

This report shows that selective mesenteric arterial infusion of vasopressin may be successful in the management of massive lower gastrointestinal bleeding in Crohn's disease.

\section{References}

1 Homan WP, Tang CK, Thorbjarnarson B. Acute massive haemorrhage from intestinal Crohn's disease: Report of seven cases and review of the literature. Arch Surg 1979; 111: 901-5.

2 Harvey JC, Rotstein L, Steinherdt M, Reingold MM, Rubin E, Stone RM. Massive lower gastrointestinal bleeding: an unusual complication of Crohn's disease. Can J Surg 1978; 21: 444-5.

3 Podolny GA. Crohn's Disease presenting with massive lower gastrointestinal haemorrhage. Am J Roentgenol 1978; 130: 368-70.

4 Nusbaum M, Baum S, Blakemore WS, Tumen $H$. Clinical experience with selective intra-arterial infusion of vasopressin in the control of gastrointestinal bleeding from arterial sources. Am J Surg 1972; 123: 165-72.

5 Nusbaum M, Baum S. Radiographic demonstration of unkown sites of gastrointestinal bleeding. Surg Forum 1963; 14: 374-5.

6 Ring EJ, Athanasoulis CA, Wattman AC. The pseudovein: an angiographic appearance of arterial haemorrhage. J Can Assoc Radiol 1973; 24: 242-44.

7 Erickson U, Fagerberg S, Krause U. Angiographic studies in Crohn's disease and ulcerative colitis. Am J Roentgenol 1970; 110: 385-92.

8 Gordon RL, Ring EJ, Baum S. The present status of angiographic diagnosis and treatment of gastrointestinal bleeding. J C E Radiol 1979; 1: 35-49. 